\title{
Evans function stability of non-adiabatic combustion waves
}

\author{
By V. V. Gubernovt, G. N. Mercer, H. S. Sidhu and R. O. Weber \\ School of Physical, Environmental and Mathematical Sciences, University of \\ New South Wales at the Australian Defence Force Academy, Canberra ACT 2600, \\ Australia (g.mercer@adfa.edu.au; h.sidhu@adfa.edu.au; r.weber@adfa.edu.au)
}

Received 22 August 2003; revised 5 December 2003; accepted 16 January 2004; published online 18 May 2004

In this paper we investigate the linear stability and properties of the planar travelling non-adiabatic combustion front for the cases of zero and non-zero ambient temperature. The speed of the front is estimated numerically using the shooting and relaxation methods. It is shown that for given parameter values the solution either does not exist or there are two solutions with different values of the front speed, which are referred to as 'fast' and 'slow'. The Evans function approach extended by the compound-matrix method is employed to numerically solve the linear-stability problem for the travelling-wave solution. We demonstrate that the 'slow' branch of the solutions is unstable, whereas the 'fast' branch can be stable or exhibits Hopf or Bogdanov-Takens instability, depending on the parameter values.

Keywords: combustion waves; Evans function; heat loss; ambient temperature; pulsating instabilities

\section{Introduction}

The reaction-diffusion models describing the propagation of combustion waves have been the subject of study for a long time (Merzhanov \& Rumanov 1999). Much effort has been invested into understanding the properties of the stationary propagating fronts and their stability. The main focus of this paper is the investigation of the properties and stability of the steady planar combustion waves with respect to pulsating (longitudinal) perturbations. This allows a one-dimensional (1D) formulation of the propagation problem and is convenient for both analytical and numerical treatment. It is also a physically reasonable problem to investigate, since pulsating instabilities represent a distinct mechanism for the loss of stability of the travelling-wave solution (Merzhanov \& Rumanov 1999). Indeed, as shown in Margolis \& Matkowsky (1983) and Margolis (1991), the geometrical parameters of the system can be chosen in such a way that the transverse instabilities can be excluded. Moreover, pulsating and cellular instabilities manifest themselves in different disjoint domains in the space of the reaction parameters (Merzhanov \& Rumanov 1999).

\footnotetext{
$\dagger$ Present address: International Laser Center, M. V. Lomonosov Moscow State University, Moscow 119899, Russia (vvg@quantum.phys.msu.su).
}

Proc. R. Soc. Lond. A (2004) 460, 2415-2435

(C) 2004 The Royal Society 
Namely, pulsating instabilities have been observed for the values of the Lewis number $(L e)$ greater than 1, whereas cellular instabilities are known to appear for the values of the Lewis number smaller than 1 (Merzhanov \& Rumanov 1999). For this reason, the mechanism leading to cellular waves is also excluded by an appropriate choice of the parameters (i.e. for a Lewis number greater than 1, which is the case in this paper). Finally, the experimental observation of the pulsating waves in selfpropagating high-temperature synthesis (SHS) (Moore \& Feng 1995a, b; Merzhanov \& Rumanov 1999; Makino 2001) is probably the best evidence that, under certain conditions, longitudinal instabilities are the dominant way in which a planar wave can lose its stability. As noted in Makino (2001) pulsating combustion frequently occurs in experiments on SHS leading to a layered structure of the resulting materials, which is often an undesirable effect. This makes the study and control of the transition from steady to pulsating combustion a subject of great practical importance.

Consideration of the propagation problem in 1D configuration, which is used throughout this paper, is not new. It has been used in a number of papers to predict and to investigate phenomena such as pulsating waves (Matkowsky \& Sivashinsky 1978; Matkowsky \& Olagunju 1980; Margolis 1991), period doubling in the oscillations of speed (Shkadinskii et al. 1971; Weber et al. 1997) and chaotic flames (Bayliss \& Matkowsky 1990; Margolis 1991; Brailovsky \& Sivashinsky 1993; Frankel et al. 1994).

The standard model describing the propagation of combustion waves involves the reaction-diffusion equations for two components: the temperature, and the amount of fuel, with Arrhenius reaction terms giving the strong nonlinear dependence of the reaction rate on the temperature (Margolis \& Matkowsky 1983; Schult 1999). For the sake of simplicity, the effect of heat exchange with the media surrounding the system is usually neglected.

The analytical investigation of the adiabatic case is based upon the application of the matched asymptotic expansion (MAE) method, which allows one to describe the properties of the steady solution, such as speed, in a consistent way in the limit of large activation energy (Bush \& Fendell 1970; Margolis \& Matkowsky 1983). However, the analysis of the linear-stability problem using the MAE method encounters difficulties known as the closure problem (Margolis \& Matkowsky 1983; Schult 1999). There are several different ways to circumvent this, including the truncated series or using the nearly equidiffusional approximation (Margolis \& Matkowsky 1983). The latter method requires the Lewis number to be asymptotically close to unity, whereas the truncated models were derived for general values of the Lewis number. The systems described above are usually investigated by numerically solving the partial differential equations (PDEs) (Weber et al. 1997). This allows one to analyse both the stationary solution and its stability. However, it requires sufficiently large computational resources in comparison with the formulation of the problem using ordinary differential equations (ODEs). In addition, the ODE formulation of the problem has the advantage of being more accurate and providing more details about the system under consideration. In Gubernov et al. (2003) the properties of the steady adiabatic front were investigated and the linear-stability problem was solved in the ODE formulation using the Evans function method. This approach has been previously applied to different nonlinear PDE stability analysis (Sandstede 2002).

Proc. R. Soc. Lond. A (2004) 
However, the adiabatic approximation narrows the applicability of the model in the real world, where taking into consideration the heat loss sometimes seems to be essential. In the present work we address a long-standing problem (Joulin \& Clavin 1979; Booty et al. 1987), which includes the effect of heat exchange with the surroundings on the propagation of combustion waves. One of the main differences between the adiabatic and non-adiabatic approximation is that the stationary solution does not always exist. The values of the heat loss for which the solution exists are relatively small in comparison with other terms in the governing equations and the temperature of the travelling front decays very slowly in the product zone. For analytical investigation using the MAE in the limit of high activation energy, this means that in order to take into account the effects of the heat loss, we cannot truncate the expansion on the leading-order terms (as was done in the adiabatic case), but we have to consider higher-order terms as well (see Joulin \& Clavin (1979), where the properties of the stationary solution were studied for arbitrary values of the Lewis number). Nevertheless, the residual amount of fuel left behind the front cannot be found using the MAE in the limit of high activation energy. However, this difficulty was overcome in the opposite limit of strongly exothermic combustion waves (Billingham \& Mercer 2001). For the linear-stability analysis the closure problem can be circumvented only in the nearly equidiffusional limit (Joulin \& Clavin 1979). For the general parameter values both the steady solution and its stability can only be investigated numerically.

The numerical analysis of the problem in the PDE formulation (Mercer et al. 1998) encounters serious obstacles. Firstly, it is very difficult to follow the solution profile in the product zone and, therefore, the dependence of the residual amount of fuel on the parameters of the problem is not easy to ascertain. Secondly, PDE methods are unable to investigate the stationary solutions once they have become unstable. In this paper we take advantage of the ODE formulation of the problem, which is usually more convenient for numerical analysis. Besides the benefits of technical implementation, an ODE formulation does not depend on the stability of the travelling wave and therefore allows us to continue the solution over a broader parameter range (including the investigation of the slow solution branch, which is impossible in the PDE formulation). Also, using the ODE approach we are able to investigate the dependence of the residual amount of fuel on the system parameters. To the best of our knowledge, this has not been done for moderate and large values of the activation energy neither analytically, using MAE, nor numerically, employing the PDE integration, for the reasons mentioned above.

The numerical-stability analysis was carried out previously by direct integration of the governing PDEs (Mercer et al. 1998). This method can be used in order to determine the neutral stability boundary for the steady wave. However, as is noted in Gubernov et al. (2003), we cannot expect this method to be accurate near the critical parameter values, where the rate of instability is weak and a long integration time is needed to detect it. This is especially true for the monotonic scenario of transition to instability (in contrast to the oscillatory scenario via Hopf bifurcation). In addition to this, PDE methods are unable to investigate the stationary solutions once they have become unstable. Therefore, the information about the behaviour of eigenvalues in the right half-plane after the transition to instability cannot be investigated using this approach. The examination of the eigenvalues of the unstable steady solution is of interest, since it can indicate the Bogdanov-Takens bifurcation, which explains the transition from the onset of monotonous to oscillating instabilities. This bifurca- 
tion was predicted analytically by means of MAE (Joulin \& Clavin 1979). However, the MAE approach is only valid for asymptotically large values of the activation energy and values of the Lewis number asymptotically close to unity. Of interest here is to investigate the behaviour of eigenvalues in the right half-plane for moderate values of parameters. This study was conducted in the present paper by using the Evans function approach, developed in Gubernov et al. (2003) for the adiabatic case. However, this method cannot be implemented directly for the problem under investigation, since the heat exchange with the surroundings imposes additional technical difficulties. The difference in the characteristic coordinate length, in which the solution changes significantly in the preheat, reaction and product zones is substantially larger in the non-adiabatic case than it was in the model without heat loss considered in Gubernov et al. (2003). Therefore, all the numerical algorithms have been modified to take into account this difference by using a non-uniform coordinate grid.

We note here that, strictly speaking, the presence of the zero eigenvalue due to the shift symmetry violates the assumptions of the classical Hopf and BogdanovTakens bifurcation theorems. This degeneracy requires a special study and lies beyond the scope of this paper. However, here we use the terms Hopf and BogdanovTakens bifurcation following the standard practice in combustion literature (Bayliss \& Matkowsky 1990; Brailovsky \& Sivashinsky 1993; Frankel et al. 1994).

Inclusion of the heat loss in the model makes it more realistic; however, one of the most straightforward control parameters in experiments is the ambient temperature. The effect of the ambient temperature on combustion processes is usually investigated when considering ignition problems (Weber et al. 1998; Watt et al. 1999). However, this issue has received little attention in the context of propagation problems, although there are experimental data showing the effect of the variation of the ambient temperature on the properties and the stability of the steady planar combustion waves in SHS (Moore \& Feng 1995a,b; Makino 2001). Ambient temperature is implicitly included in parameters for many combustion studies via the Zeldovich number. Analytical investigation of the flame propagation usually uses asymptotic methods, which require the Zeldovich number to be asymptotically large (Schult 1999). As a result the ambient temperature does not appear in the final results of the asymptotic analysis. On the other hand, the standard choice of nondimensional parameters is not convenient for numerical studies either and, to the best of our knowledge, the effect of the ambient temperature on the propagation of combustion waves has not been systematically investigated by means of numerical analysis. In this paper, following Weber et al. (1997), we use a model which combines the ambient temperature with other dimensional parameters in a manner that makes the effect of the ambient temperature explicit. This allows us to carry out the numerical investigation of the effect of the ambient temperature on propagation of combustion waves.

This paper is essentially divided into two parts. In the first part we investigate the properties of the stationary propagating solution numerically employing the shooting and relaxation methods. The linear-stability analysis is the focus of the second part. In both of these parts, we examine the behaviour of the system for zero and non-zero ambient temperature. We use the Evans function method (extended by the compound-matrix method) to determine the stability of the non-adiabatic combustion fronts.

Proc. R. Soc. Lond. A (2004) 


\section{Model}

We consider a premixed fuel in one dimension. It is assumed that the rate of exothermic combustion is well described by the Arrhenius law. In non-dimensional coordinates, the equations governing this process can be found in Weber et al. (1997) and are given as

$$
u_{t}=u_{x x}+v \mathrm{e}^{-1 / u}-l\left(u-u_{\mathrm{a}}\right), \quad v_{t}=\tau v_{x x}-\beta v \mathrm{e}^{-1 / u},
$$

where $u$ and $v$ are non-dimensional temperature and the mass fraction of fuel, respectively, $\tau$ is the inverse Lewis number (the ratio of the diffusion rates of mass and heat), $\beta$ is the ratio of the activation energy to heat release, $l$ is the heat-loss coefficient from fuel to surroundings and $u_{\mathrm{a}}$ is the ambient temperature.

We consider the ambient temperature to be a small quantity in the current nondimensional variables. This approximation is quite feasible for propagation problems. In experiments the dimensionless ambient temperature usually varies between 0 and 0.03 (Makino 2001). Parameter $\tau$ varies from $0(L e \rightarrow \infty)$ for solid fuel, to unity $(L e=1)$ for gaseous fuels. The parameter $\beta$ is of the order of unity or larger. The heat loss $l$ is a parameter which can be manipulated in the laboratory. In order for stationary solutions to exist, $l$ must be taken to be sufficiently small, as will be seen in the next section.

We consider system (2.1) subject to the following boundary conditions.

$$
\begin{aligned}
& u(x, t) \rightarrow u_{\mathrm{a}}, \quad v_{x}(x, t) \rightarrow 0, \quad \text { as } x \rightarrow-\infty, \\
& u(x, t) \rightarrow u_{\mathrm{a}}, \quad v(x, t) \rightarrow 1, \quad \text { as } x \rightarrow+\infty .
\end{aligned}
$$

On the right boundary we have a cold $\left(u=u_{\mathrm{a}}\right)$ and unburned $(v=1)$ state, whereas the opposite limit corresponds to a partly burned $(v=\sigma)$ state, where the temperature is cooling down to the ambient value $\left(u=u_{\mathrm{a}}\right)$. Here $\sigma$ is the residual amount of fuel left after the combustion wave ( $\sigma=0$ for the adiabatic case).

\section{Travelling-wave solution}

In this section we investigate the properties of the travelling solution. We first study the case where $u_{\mathrm{a}}=0$ and then we show how the non-zero ambient temperature alters these results.

(a) Zero ambient temperature $\left(u_{\mathrm{a}}=0\right)$

We will seek the solution of (2.1) in a form of the front travelling with a constant speed $c$

$$
u(x, t)=u(\xi), \quad v(x, t)=v(\xi),
$$

where we have introduced a moving coordinate frame $\xi=x-c t$. After substituting (3.1) into (2.1) it is easy to obtain two second-order differential equations

$$
u_{\xi \xi}+c u_{\xi}+v \mathrm{e}^{-1 / u}-l u=0, \quad \tau v_{\xi \xi}+c v_{\xi}-\beta v \mathrm{e}^{-1 / u}=0,
$$

and boundary conditions

$$
\left.\begin{array}{l}
u=0, \quad v_{\xi}=0, \quad \text { as } \xi \rightarrow-\infty, \\
u=0, \quad v=1, \quad \text { as } x \rightarrow+\infty .
\end{array}\right\}
$$


Equations similar to (3.2), (3.3) were considered analytically in Joulin \& Clavin (1979) based on the MAE method with $\beta^{-1}$ as a small parameter. It was assumed that all the fuel had been consumed and that there was no reaction in the product zone. In the second order of the asymptotic procedure, the following expression for the speed of the front was obtained

$$
c^{2}=c_{\mathrm{ad}}^{2} \mathrm{e}^{-2 \beta l / c^{2}}
$$

where

$$
c_{\mathrm{ad}}=\sqrt{\frac{2}{\tau \beta}} \mathrm{e}^{-\beta / 2}
$$

is the adiabatic flame speed $(l=0)$, which can be derived in the first order of the MAE (Margolis \& Matkowsky 1983). The flame speed estimation (3.4) is valid for arbitrary values of $\tau$ (the inverse Lewis number) and $\beta \gg 1$. The formula for a speed of the front (3.4) reveals the drastic difference in comparison with the adiabatic problem. Namely, for fixed parameter $\tau$ there is a critical value of $\beta_{\mathrm{e}} l_{\mathrm{e}}$ such that the steady propagating front solutions exist for $\beta l$ less than the critical value, and do not exist for greater values of $\beta l$. This effect is called extinction (sometimes this event is called a saddle-node or a blue-sky bifurcation or a turning point) and we use subscript 'e' for the notation of the critical-parameter values, which can be found from (3.4) by solving the equation

$$
\frac{\partial(\beta l)}{\partial c}=2 c \ln \left(\frac{c_{\mathrm{ad}}}{c}\right)-c=0 .
$$

This results in the following equation for the critical heat loss

$$
l_{\mathrm{e}}(\beta, \tau)=\frac{\mathrm{e}^{-1-\beta}}{\tau \beta^{2}} .
$$

For fixed values of $\beta, \tau$ and $l<l_{\mathrm{e}}$ there exist two solutions with different speed, a 'fast' and a 'slow' solution.

We solve equations (3.2), (3.3) numerically by using the shooting method to obtain the guess solution and then the results are corrected with a more accurate method, namely, relaxation. However, as we have mentioned above, there is a great difference between numerical integration of the adiabatic and non-adiabatic problems. It follows from (3.7) that the solution exists only if the heat loss is sufficiently small. On the other hand, in the product zone the rate of the exponential decay of the temperature to zero is governed by $l$. This implies that in order to determine the stationary front numerically one has to follow the slowly decaying tails in the product zone, followed by a very rapid jump in the temperature and the amount of fuel in the reaction zone, and finally relatively fast decay in the preheat zone. Therefore, in contrast to the adiabatic problem (Gubernov et al. 2003), we use a non-uniform mesh along the $l<l_{\mathrm{e}}$ coordinate. The fifth-order Runge-Kutta method with the adaptive stepsize control is employed for the shooting method. The method not only allows the estimation of the local error, which has been set to be $10^{-5}$ in our calculations, but at the same time it produces the non-uniform grid on the interval of integration. The relaxation method has been modified in order to deal with the non-uniform grids. The stability analysis of the steady propagating combustion front carried out in the following sections is based on the accuracy of our approximation of the solution to 


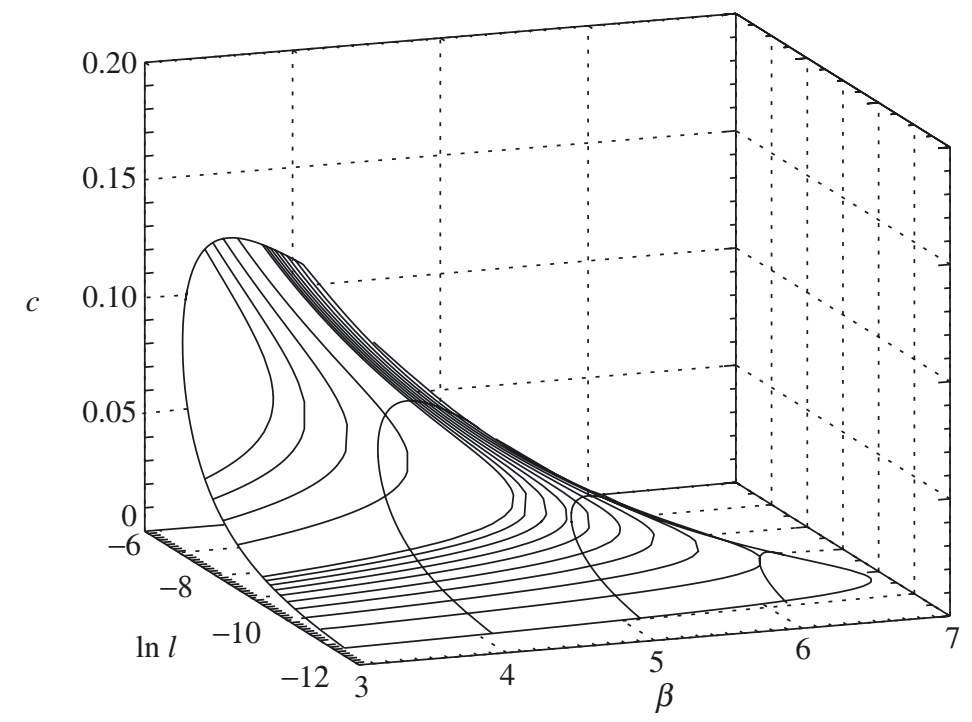

Figure 1. Numerically determined speed of the travelling front as a function of $\beta$ and $\ln l$ for $\tau=0.5$.

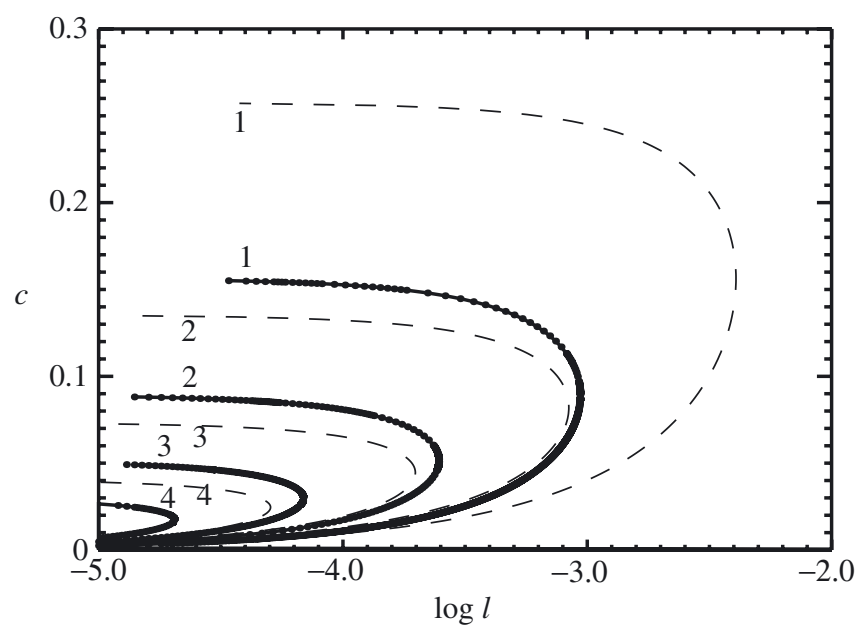

Figure 2. Speed of the travelling front as a function of $l$ for $\tau=0.5$ and four different values of $\beta$ : curves 1 correspond to $\beta=3.0$, curves 2 to $\beta=4.0$, curves 3 to $\beta=5.0$, and curves 4 to $\beta=6.0$. Dots connected with solid lines represent the numerical results obtained with shooting and relaxation methods, whereas dashed lines were plotted according to the formula (3.4).

equation (3.2). The relaxation routine allows us to control the average local correction made on each iteration step. The solution is considered to be resolved if the correction is less than $10^{-15}$. In figure 1 we plot the dependence of the speed of the front on $\beta$ and $l$ for fixed value of $\tau=0.5$. The surface looks like a bent page and has a clear edge viewed from the outermost corner of the coordinate box. In figure 2 we plot several cross-sections of the surface $c(\beta, l)$ by the planes $\beta=3,4,5,6$. We see that for fixed $\beta$ and some value of $l_{\mathrm{e}}$ the derivative $\partial c / \partial l$ becomes infinite. This $l_{\mathrm{e}}$ corresponds to extinction. There are two stationary propagating fronts for $l<l_{\mathrm{e}}$ and 


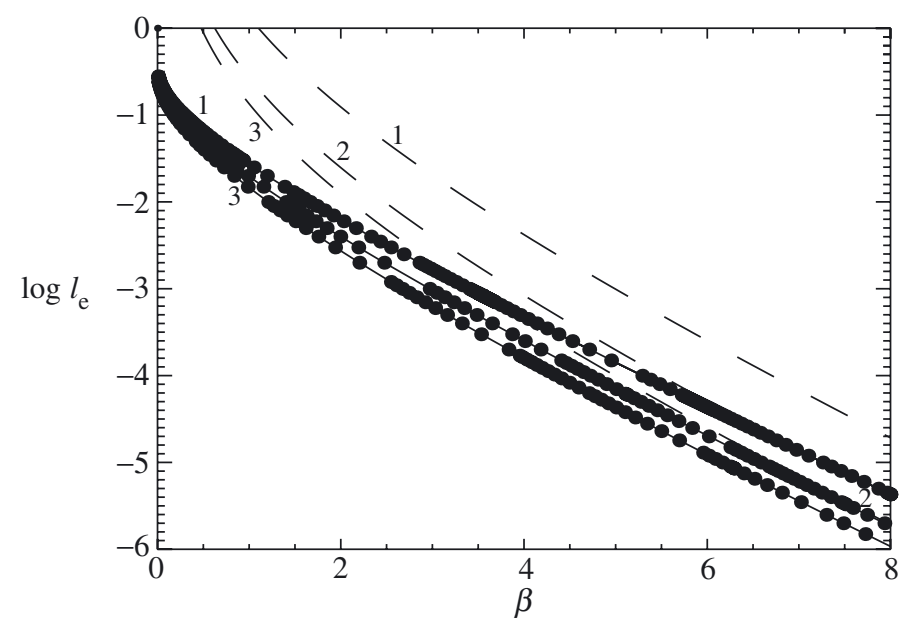

Figure 3. Dependence of the extinction value of $l$ on $\beta$ for different values of $\tau$ : curves 1 for $\tau=0.1$, curves 2 for $\tau=0.5$, and curves 3 for $\tau=1.0$. Dots connected with solid lines correspond to numerical results. Dashed lines were plotted according to (3.7).

there are no solutions for $l>l_{\mathrm{e}}$. We have also plotted the prediction of the asymptotic formula (3.4) on the same figure. As can be seen the results obtained from the MAE method provide only qualitative behaviour of the system and the parameter values at the point of extinction are poorly approximated by (3.7). In figure 3 we plot the extinction values of heat loss as a function of $\beta$ for several values of $\tau$. The numerical results have been obtained by solving the equation $\partial \beta / \partial c=0$ using the NewtonRaphson method for fixed $l$. The critical value of $\beta$ is considered to be resolved if the derivative is less than $10^{-8}$. The difference between the numerical data and the prediction of (3.7) is substantial for small and moderate values of $\beta$. For large values of $\beta$ the discrepancy is small, especially when $\tau=1$. This is expected since the MAE is valid for large values of $\beta$ and higher-order terms of the expansion would need to be considered to give a better correspondence with the numerical results.

Finally, in figure 4 the dependence of the residual amount of fuel $\sigma$ on $l$ is presented for different values of $\beta$. The larger values of $\sigma$ correspond to the slow branch of the solution for each curve. It should be noted that according to the analysis in Joulin \& Clavin (1979) the residual amount of fuel left behind the front can be neglected. From figure 4, this can be done for the fast branch; however, $\sigma$ becomes crucial when considering slow solutions.

\section{(b) Non-zero ambient temperature $\left(u_{\mathrm{a}}>0\right)$}

Next we apply the numerical methods described earlier, to investigate the effect of ambient temperature on the system and compare the results with the $u_{\mathrm{a}}=0$ case.

Firstly, we investigate the dependence of the speed of the front on the ambient temperature. In figure 5 the flame speed is shown as a function of the logarithm of the heat loss for fixed values of $\beta$ and $\tau$ and different values of $u_{\mathrm{a}}$, as indicated in the caption. As we increase the ambient temperature for fixed values of $\beta, \tau$, and $l$ the speed of the front rises. This result is in qualitative agreement with the experimental data (Makino 2001). It is interesting to note that as we increase $u_{\mathrm{a}}$ the 


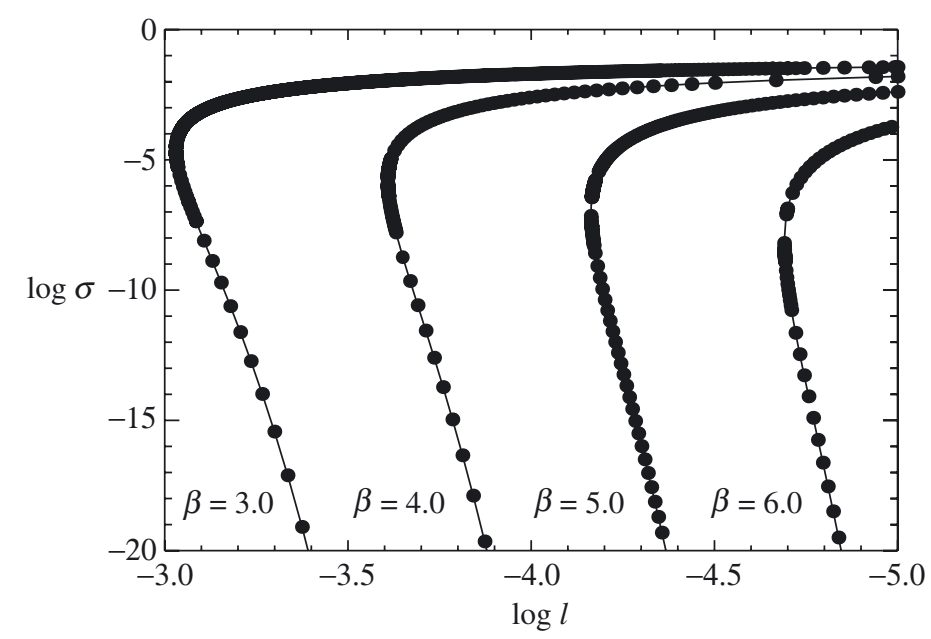

Figure 4. Numerically determined residual amount of fuel behind the reaction front as a function of $l$ for $\tau=0.5$ and four different values of $\beta=3,4,5,6$.

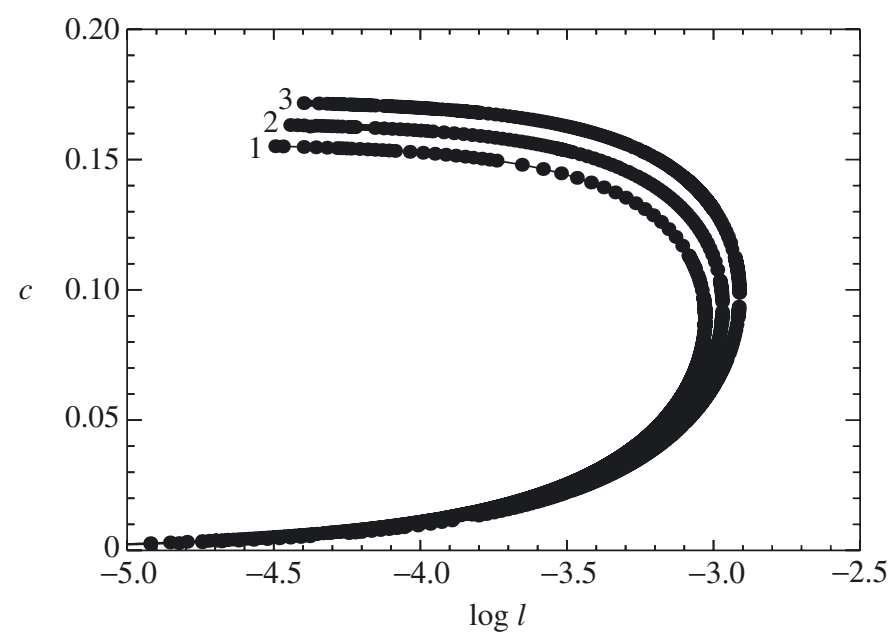

Figure 5. Speed of the travelling front as a function of $l$ for $\tau=0.5, \beta=3$ and different values of $u_{\mathrm{a}}$ : curve 1 corresponds to $u_{\mathrm{a}}=0$, curve 2 to $u_{\mathrm{a}}=0.005$, curve 3 to $u_{\mathrm{a}}=0.01$. These results were obtained with shooting and relaxation methods.

point of extinction moves towards larger values of heat loss resulting in an increase in the region of parameter values for which the travelling-wave solution exists. This is clearly seen in figure 6 , where we plot the extinction values of heat loss as a function of $\beta$ for $\tau=0.5$ and for different values of $u_{\mathrm{a}}$.

It is clear from the non-zero ambient temperature results that the overall qualitative behaviour is the same as for the zero ambient temperature approximation that is often used to circumvent the so-called cold boundary problem. For problems where quantitative results are required obviously a non-zero ambient temperature is needed.

Next we proceed to the investigation of the stability of the travelling combustion wave.

Proc. R. Soc. Lond. A (2004) 


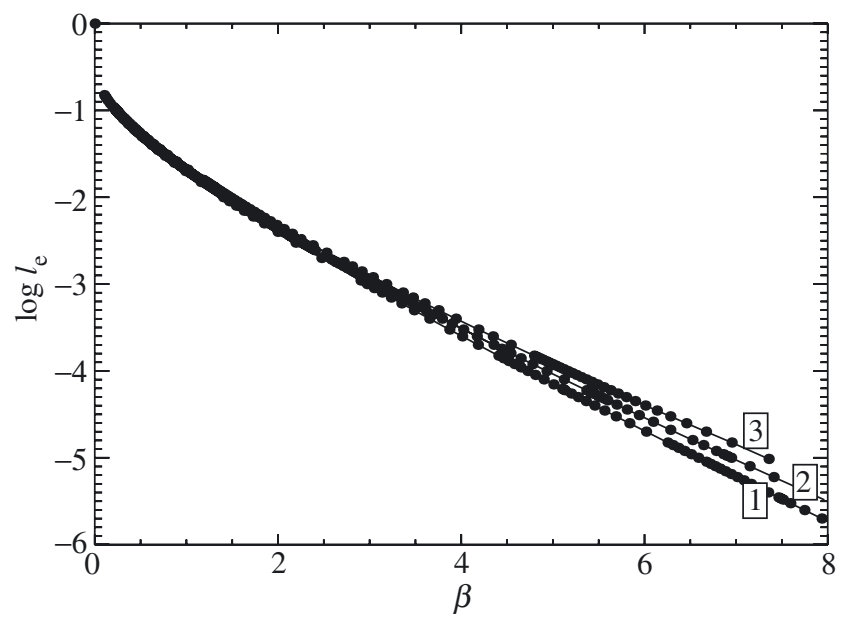

Figure 6. Dependence of the extinction value of $l$ on $\beta$ for $\tau=0.5$ and different values of $u_{\mathrm{a}}$ : curve 1 for $u_{\mathrm{a}}=0$, curve 2 for $u_{\mathrm{a}}=0.005$ and curve 3 for $u_{\mathrm{a}}=0.01$. These results were obtained numerically.

\section{Stability of the travelling front}

As a first step in the analysis of the travelling-wave stability we linearize (2.1) around the front solution (3.1)

$$
u(x, t)=u(\xi)+\varphi(\xi, t), \quad v(x, t)=v(\xi)+\chi(\xi, t),
$$

where $\varphi$ and $\chi$ are linear perturbation terms. After substitution of (4.1) into (2.1) it is straightforward to derive

$$
\left(\begin{array}{l}
\partial \varphi / \partial t \\
\partial \chi / \partial t
\end{array}\right)=\hat{L}\left(\begin{array}{l}
\varphi \\
\chi
\end{array}\right)
$$

where

$$
\hat{L}=\left(\begin{array}{cc}
\partial_{\xi}^{2}+v u^{-2} \mathrm{e}^{-1 / u}+c \partial_{\xi}-l & \mathrm{e}^{-1 / u} \\
-\beta v u^{-2} \mathrm{e}^{-1 / u} & \tau \partial_{\xi}^{2}-\beta \mathrm{e}^{-1 / u}+c \partial_{\xi}
\end{array}\right) .
$$

The stability of the travelling front is then defined from the spectrum of $\hat{L}$. It is easy to show that the essential spectrum of this operator always lies in the left half-plane (see Henry 1981; Volpert et al. 1994). In order to do this we consider the limiting operators $\hat{L}^{ \pm}=\lim _{\xi \rightarrow \pm \infty} \hat{L}$. It can be shown that the essential spectrum of $\hat{L}^{ \pm}$consists of algebraic curves

$$
\lambda_{1}(k)=-k^{2}+\mathrm{i} c k-l, \quad \lambda_{2}(k)=-\tau k^{2}+\mathrm{i} c k,
$$

where $k \in(-\infty,+\infty)$, which are located in the left half-plane symmetric about the real axis. The second curve in (4.4) includes the origin. Suppose that $K$ is the union of the regions inside or on these curves, so that $\mathbb{C} \backslash K$ contains the right half-plane. Then, according to Henry (1981), the essential spectrum of $\hat{L}$ is contained in $K$, and in particular includes curves (4.4). Therefore, the essential spectrum of $\hat{L}$ lies in the left half-plane (including the origin) and the discrete spectrum is solely responsible for the transition to instability. We will seek the solution of (4.2) of the form

$$
\varphi(\xi, t)=\varphi(\xi) \mathrm{e}^{\lambda t}, \quad \chi(\xi, t)=\chi(\xi) \mathrm{e}^{\lambda t},
$$

Proc. R. Soc. Lond. A (2004) 
where $\lambda$ is a spectral parameter (in combustion literature it is sometimes referred to as the growth-rate eigenvalue). Substituting (4.5) into (4.2) and introducing a vector with components $z_{1}=\varphi, z_{2}=\varphi_{\xi}, z_{3}=\chi, z_{4}=\chi_{\xi}$, we obtain a system of ODEs in the form

$$
\dot{z}=A z
$$

where

$$
\boldsymbol{A}(\xi, \lambda)=\left(\begin{array}{cccc}
0 & 1 & 0 & 0 \\
\lambda+l-v u^{-2} \mathrm{e}^{-1 / u} & -c & -\mathrm{e}^{-1 / u} & 0 \\
0 & 0 & 0 & 1 \\
\beta \tau^{-1} v u^{-2} \mathrm{e}^{-1 / u} & 0 & \tau^{-1}\left(\lambda+\beta \mathrm{e}^{-1 / u}\right) & -\tau^{-1} c
\end{array}\right)
$$

We use equation (4.6) to investigate the stability of the travelling front. Following Afendikov \& Bridges (2001), we will say that the travelling front is linearly unstable if, for some fixed complex $\lambda$ with $\operatorname{Re}(\lambda)>0$, there exists a solution of (4.6) which decays exponentially as $\xi \rightarrow \pm \infty$. We will refer to this $\lambda$ as an eigenvalue and to the corresponding solution as an eigenmode.

\section{Matched asymptotic analysis for the linear-stability problem $\left(u_{\mathrm{a}}=0\right)$}

The linear-stability problem (4.2), (4.3) is considered in Joulin \& Clavin (1979) and Booty et al. (1987) in the framework of the MAE method. We would like to briefly quote the main results which are relevant to our work here.

The solution to (4.2), (4.3) together with the parameters of the system is sought in the form of the series with $\beta^{-1}$ being a small parameter of the asymptotic procedure. Substituting these expansions into (4.2), (4.3) and collecting terms of the same order as the small parameter we can obtain a separate problem in the leading order, first order, etc. However, it is immediately evident that these equations result in the so-called closure problem. We see that the leading-order problem is related to the first-order problem. Similarly, the solution of the first-order problem will depend on the second-order problem, etc. In other words, to find the leading-order solution we have to investigate the infinite number of problems arising in each step of the asymptotic expansion.

To circumvent the closure problem for the adiabatic system the truncation of the series is made in such a way so as to take into account only the leading-order terms of the asymptotic expansion (Margolis \& Matkowsky 1983). However, for the non-adiabatic case this approach is not applicable, since the heat-loss term manifests itself only in the first order of the expansion. This implies that the first-order problem has to be solved. However, the solution of this problem is by itself challenging and is subject to technical difficulties. To the best of our knowledge it has not been considered in the relevant literature.

The only way to circumvent the closure problem is to put $\tau_{0}=1$ (nearly equidiffusional) following Joulin \& Clavin (1979) and Booty et al. (1987). In this case the equation relating the leading-order spectral parameter with the parameters of the problem can be derived, namely

$$
\Gamma^{2}-h(\Gamma+1)=\frac{1}{4} \tau_{1}(1-\Gamma),
$$

Proc. R. Soc. Lond. A (2004) 
where $\Gamma=\sqrt{1+4 \lambda}, h=\beta l c^{-2}$, and $\tau_{1}$ is the first-order term in the asymptotic expansion $\tau=1+\beta^{-1} \tau_{1}+\cdots$. It is worthwhile to note that the point of extinction corresponds to $h=1 / 2$. Then $h<1 / 2$ corresponds to the fast branch, whereas $h>1 / 2$ refers to the slow branch. Simple algebraic manipulation of (5.1) shows that there is always one solution with $\operatorname{Im}(\lambda)=0$ and $\operatorname{Re}(\lambda)>0$ for the slow branch $(h>1 / 2)$, therefore the slow fronts are always unstable (or in other words, a single point of the discrete spectrum located on the right half-plane). When $-6<\tau_{1}<0$ this point moves along the real axis and crosses the origin at the point of extinction ( $h=1 / 2$ ) as we go from the slow to the fast branch, so that the fast fronts are stable for $-6<\tau_{1}<0$. When $\tau_{1}<-6$ the system exhibits a different bifurcation, namely, that as we move along the slow branch $(h>1 / 2)$ towards the extinction limit $(h=1 / 2)$, another point of discrete spectrum moves along the real axis from the left half-plane to the right half-plane, and it crosses the origin at the critical values of parameters, corresponding to the extinction (3.7). Immediately after passing from the slow to the fast branch, there are two points of discrete spectrum in the right half-plane, both located on the real axis, and the solution is unstable. Next, as we change the parameters of the system so as to remain on the fast branch, a pair of points of the discrete spectrum collides when the line $h_{1}\left(\tau_{1}\right)=-2+\tau_{1} / 4+\sqrt{4-2 \tau_{1}}$ is crossed in the parameter space. After the collision $h<h_{1}$ the points of discrete spectrum diverge symmetrically from the real axis, giving rise to oscillatory instability. Finally, when $h_{2}\left(\tau_{1}\right)=\tau_{1} / 4-1+\sqrt{3-\tau_{1}}$ is crossed, the system exhibits Hopf bifurcation and the solution becomes stable for $h<h_{2}$. It is important to note here (and this was mentioned earlier) that, although this is not exactly the classical Hopf bifurcation, due to the presence of the zero eigenvalue as a result of shift symmetry, in combustion literature it is often still termed Hopf bifurcation, since it results in oscillatory solutions.

\section{Evans function method for the linear-stability problem}

In this section we return to the linear-stability problem (4.6), (4.7). Let us introduce the limit matrix

$$
\boldsymbol{A}(\lambda) \equiv \lim _{\xi \rightarrow \pm \infty} \boldsymbol{A}(\xi, \lambda) .
$$

The explicit form of $\boldsymbol{A}$ can be found from the boundary conditions (3.3). The limit matrix has eigenvalues

$$
\mu_{1,2}(\lambda)=\frac{-c \mp \sqrt{c^{2}+4 \lambda \tau}}{2 \tau}, \quad \mu_{3,4}(\lambda)=\frac{-c \mp \sqrt{c^{2}+4(\lambda+l)}}{2},
$$

with corresponding eigenvectors

$$
\boldsymbol{k}_{1,2}=\frac{1}{\sqrt{1+\mu_{1,2}^{2}}}\left(0,0,1, \mu_{1,2}\right)^{\mathrm{T}}, \quad \boldsymbol{k}_{3,4}=\frac{1}{\sqrt{1+\mu_{3,4}^{2}}}\left(1, \mu_{3,4}, 0,0\right)^{\mathrm{T}},
$$

where $\mathrm{T}$ stands for transposition. Equations (6.2) imply that $\boldsymbol{A}$ has two eigenvalues $\mu_{2,4}$ with positive real parts and two eigenvalues $\mu_{1,3}$ with negative real parts. Therefore, for any value of $\lambda$ there exist two linearly independent solutions $\boldsymbol{z}_{2,4}(\xi, \lambda)$ of (4.6) corresponding to the unstable subspace of $\boldsymbol{A}$ satisfying the conditions

$$
\lim _{\xi \rightarrow-\infty} \exp \left(-\mu_{i} \xi\right) \boldsymbol{z}_{i}(\xi, \lambda)=\boldsymbol{k}_{i}, \quad i=2,4 .
$$

Proc. R. Soc. Lond. A (2004) 
and two linearly independent solutions $\boldsymbol{z}_{1,3}(\xi, \lambda)$ of (4.6) corresponding to the stable subspace of $\boldsymbol{A}$ satisfying the conditions

$$
\lim _{\xi \rightarrow+\infty} \exp \left(-\mu_{i} \xi\right) \boldsymbol{z}_{i}(\xi, \lambda)=\boldsymbol{k}_{i}, \quad i=1,3 .
$$

In a similar manner to that described in Gubernov et al. (2003), we can consider a space of solutions of (4.6) bounded as $\xi \rightarrow-\infty$ and a space of solutions bounded as $\xi \rightarrow+\infty$. If these spaces intersect non-trivially for some value $\lambda$, then $\lambda$ is an eigenvalue. We will call the function which measures whether these spaces intersect the Evans function. Geometrically, this means that for some value of $\lambda$ and any value of coordinate $\xi$ the plane defined by the vectors $\boldsymbol{z}_{2,4}$ intersects non-trivially with the plane defined by the vectors $\boldsymbol{z}_{1,3}$. We can also say that $\lambda$ is an eigenvalue if and only if the solutions $\boldsymbol{z}_{2,4}$ and $\boldsymbol{z}_{1,3}$ are linearly dependent or, equivalently, the Wronskian evaluated on these solutions (a matrix whose columns are $\boldsymbol{z}_{2,4}(\xi)$ and $\left.\boldsymbol{z}_{1,3}(\xi)\right)$ is equal to zero. Now we define the Evans function via this Wronskian, which is evaluated for definiteness at $\xi=0$. Let $\boldsymbol{e}_{i}$ be the orthonormal basis in four-dimensional space $C^{4}$ of system (4.6) solutions. In this basis the vectors $\boldsymbol{z}_{i}$ have coordinates $\left(\boldsymbol{z}_{i, 1}, \boldsymbol{z}_{i, 2}, \boldsymbol{z}_{i, 3}, \boldsymbol{z}_{i, 4}\right)^{\mathrm{T}}$ and the Evans function is defined as

$$
D(\lambda)=\frac{1}{4} \mu_{1}^{*} \mu_{3}^{*}\left[\begin{array}{llll}
z_{21}^{-}(0, \lambda) & z_{41}^{-}(0, \lambda) & z_{11}^{+}(0, \lambda) & z_{31}^{+}(0, \lambda) \\
z_{22}^{-}(0, \lambda) & z_{42}^{-}(0, \lambda) & z_{12}^{+}(0, \lambda) & z_{32}^{+}(0, \lambda) \\
z_{23}^{-}(0, \lambda) & z_{43}^{-}(0, \lambda) & z_{13}^{+}(0, \lambda) & z_{33}^{+}(0, \lambda) \\
z_{24}^{-}(0, \lambda) & z_{44}^{-}(0, \lambda) & z_{14}^{+}(0, \lambda) & z_{34}^{+}(0, \lambda)
\end{array}\right],
$$

where star denotes complex conjugation. The coefficient in front of the determinant in (6.6) is chosen in such a way that $D(\lambda) \rightarrow 1$ as $|\lambda| \rightarrow \infty, \operatorname{Re}(\lambda)>0$.

\section{Numerical results}

The numerical method for determining the Evans function is described in detail in Gubernov et al. (2003). It uses the compound-matrix method to eliminate the stiffness of the linear-stability problem (4.6), (4.7). However, there is a difference between the algorithm employed in this paper and the algorithm described in Gubernov et al. (2003) for the adiabatic case. This is due to the non-uniform grid, which we use to approximate the stationary solution of the non-adiabatic problem (3.2). The spectral problem (4.6) is integrated numerically by means of the fifth-order RungeKutta method with the adaptive step-size control as in $\S 3$. The numerical scheme for (4.6) requires the stationary solution, which appears in the equations explicitly, to be approximated at arbitrary values of coordinate $\xi$. In other words, grids for the systems (3.2) and (4.6) do not coincide. The Neville algorithm (Press et al. 1992) for constructing the interpolation polynomial is used to find $u(\xi)$ and $v(\xi)$ for any value of $\xi$ inside of the integration interval. The Neville scheme uses the values of $u(\xi)$ and $v(\xi)$ (obtained by numerically solving (3.2)) at four grid points closest to $\xi$ and allows the control of the error made during interpolation. In our calculations this error has been of the order of $10^{-13}$. The error of the numerical integrator has been set to be $10^{-5}$, as in $\S 3$.

The stability analysis of the travelling front of (2.1) then reduces to the search for zeros of the Evans function (6.6) located in the right half-plane. To find the zeros of 
$D(\lambda)$ we apply two techniques described in Gubernov et al. (2003). First, we use the Nyquist plots, which are based on the argument principle which says that the number of zeros of an analytic function inside of a closed contour equals the number of times the image of this contour under $D(\cdot)$ winds (wraps) around the origin. This number is often called the winding number, which we denote by $N$. If we want to determine the total number of zeros located in the right half-plane, we take a contour, including the imaginary axis and a semicircle with an infinite radius, such that all points of the contour satisfy $\operatorname{Re}(\lambda) \geqslant 0$. The circular part of the contour counts for nothing because of the Evans function asymptotic behaviour (see the previous section). Once we have found the total number of zeros in the right half-plane for some fixed $\lambda, \tau$ and $l$ we obtain their approximate location by taking several contours of decreasing area including one of the zeros. Next, we solve the equation $D(\lambda)=0$ with the Newton-Raphson method, using the data obtained above to determine good initial values. Then the solution of $D(\lambda)=0$ can be continued for different values of the parameters.

Next we consider the case of zero ambient temperature and then generalize the results for $u_{\mathrm{a}}$ greater than zero.

\section{(a) Zero ambient temperature $\left(u_{\mathrm{a}}=0\right)$}

In figure 7 we plot the bifurcation diagram for fixed values of parameters $\tau=0.1$ and $l=10^{-5}$. Here it is convenient to consider $c$ as an independent parameter, and therefore the dependence of $\beta$ on $c$, for the stationary front, is a unique function, as shown in figure $7 c$. Using the technique described above we are able to find the zeros (or points of the discrete spectrum) of the equation $D(\lambda)=0$ in the complex plane. The dependence of the real part of the points of the discrete spectrum on $c$ is presented in figure $7 a$. The location of the zeros of the Evans function in the complex plane as $c$ is varied is shown in figure $7 b$. We plot the values of $\beta$ for the stationary front versus an imaginary part of the zeros of the Evans function for different values of $c$ in figure $7 d$. As we decrease the speed from $c \approx 0.033$, a pair of complex-conjugate points of the discrete spectrum moves from the right to the left half-plane. Then at some critical value of $c_{\mathrm{H}}$, shown in figure 7 by the dashed lines marked with the label 'Hopf', these points cross the imaginary axis giving rise to an oscillatory instability. If we change $c$ further, the zeros of the Evans function approach the real axis and, at some value of the speed, they collide with each other, giving birth to a pair of purely real eigenvalues. These eigenvalues move along the real axis in opposite directions as we continue to reduce $c$. One of the points of the discrete spectrum approaches the origin when the speed of the front reaches the extinction limit, shown on figure 7 by the dashed lines marked with the label 'ext'. The other eigenvalue remains in the right half-plane causing the instability of the slow branch of the stationary front solutions.

Next we investigate how the scenario described above changes when we vary parameters $\tau$ and $l$. Critical parameters of the Hopf bifurcation are traced in exactly the same way as it was done in Gubernov et al. (2003), namely, we solve the equation $\operatorname{Re}(\lambda)=0$ together with the equation for the zeros of the Evans function $D(\lambda)=0$ by using the Newton-Raphson method. In figure 8 we plot the critical values of $c$ versus $l$ for the Hopf bifurcation and extinction. The inverse Lewis number is $\tau=0.1$. We characterize the stability in each region on the plane $(c, l)$ by the winding number 

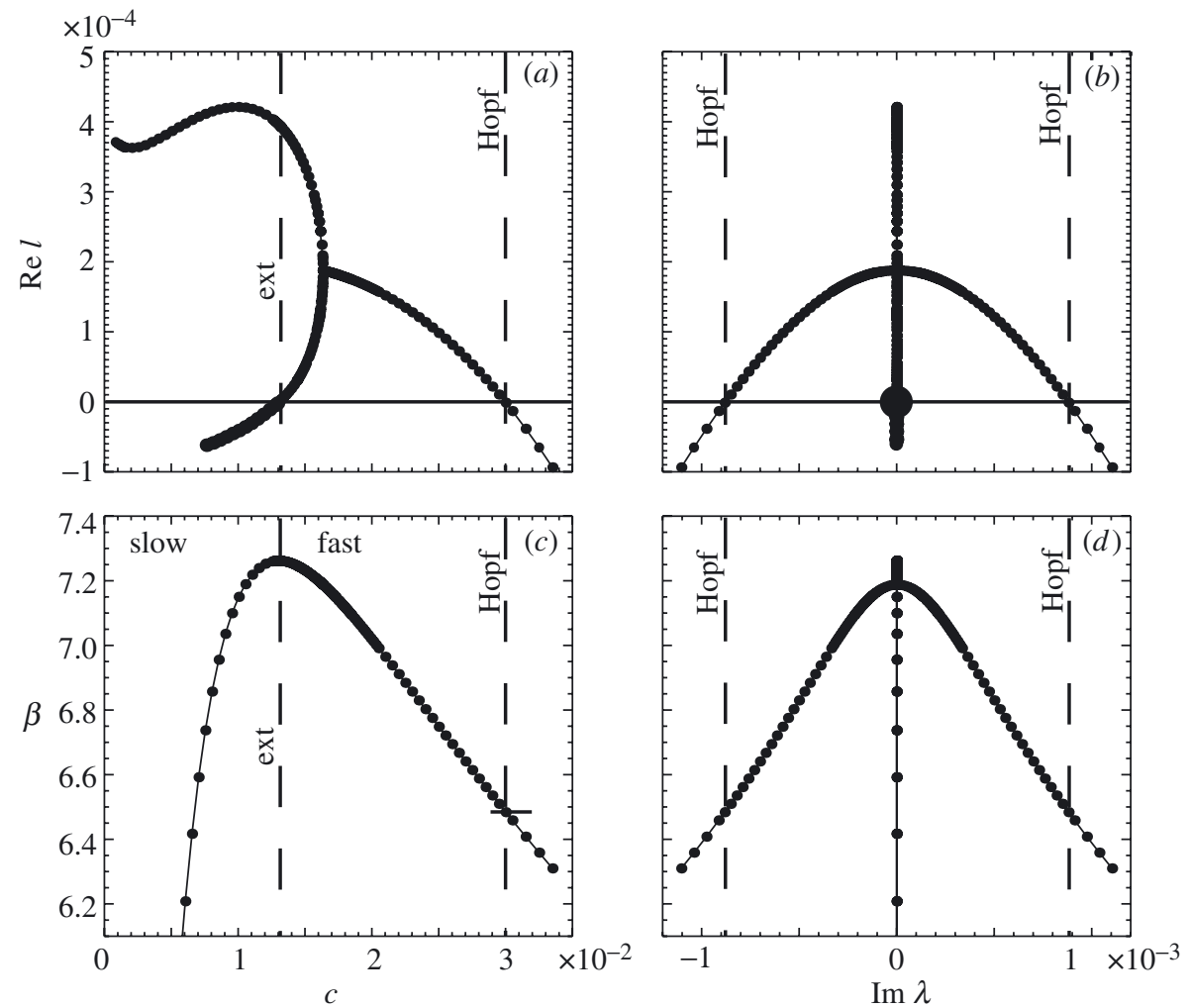

Figure 7. Bifurcation diagram for $\tau=0.1, l=10^{-5}$ and $u_{\mathrm{a}}=0$. Dots connected with solid lines correspond to numerical results. Dashed lines with the index 'Hopf' show the critical parameter values for the 'Hopf' bifurcation, whereas the index 'ext' implies extinction. ( $a$ ) The dependence of the real part of points of discrete spectrum on the speed of the front; $(b)$ the location of the points of discrete spectrum on the complex plane as we change $c ;(c)$ the dependence of $\beta$ on $c$ for the steady propagating front; $(d)$ the imaginary part of the points of discrete versus $\beta$. In part $(b)$ a circle shows zero eigenvalue, which always exists due to shift symmetry.

$N$, which is calculated using the argument principle described earlier. In region 1 the winding number $N=0$ and the solution is stable, whereas in regions 2 and 3 the winding number $N=2$ and $N=1$, respectively, indicating that the solutions are unstable. Shown in the inset is an important point 'o' on the graph, where the curve corresponding to the Hopf bifurcation intersects with the curve for the extinction. This point is sometimes called the Bogdanov-Takens bifurcation (Kuznetsov 1981). Here once again we note that this is not the classical Bogdanov-Takens bifurcation due to the presence of the zero eigenvalue. If we take $l$ less than the value at the intersection $l_{\mathrm{o}}$, then the scenario of the transition to instability as we change $c$ corresponds to the one described in the comments for figure 7 (for $l=10^{-5}$ ). As we approach the value $l_{\mathrm{o}}$ the distance between the critical speed at the points of extinction $c_{\mathrm{e}}$ and the Hopf bifurcation $c_{\mathrm{H}}$ becomes smaller until $c_{\mathrm{e}}=c_{\mathrm{H}}$ for $l=l_{\mathrm{o}}$. At this point two zeros of the Evans function move from the left half-plane to the imaginary axis, as we approach the extinction limit from above by changing the front speed (this corresponds to the change of $c$ along the dashed thin line in figure $8 a$ ). 


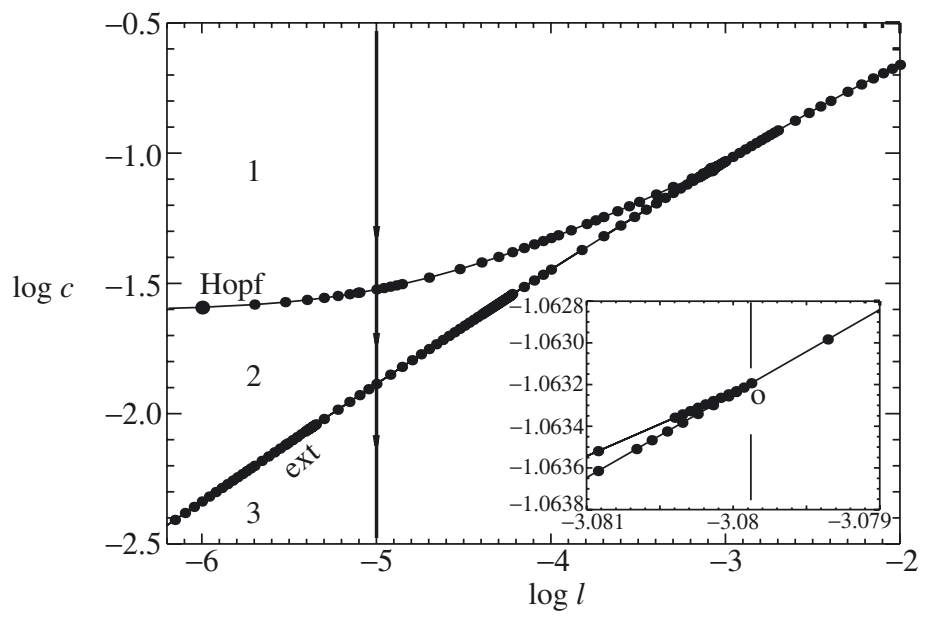

Figure 8. Critical parameter $c$ versus $l$ for Hopf bifurcation (labelled 'Hopf') and extinction (labelled 'ext') for $\tau=0.1$ and $u_{\mathrm{a}}=0$. Dots connected with solid lines correspond to numerical results. Thin line with arrows shows the path in parameter space along which we move in figure 7 . Regions 1 and 2 correspond to fast solutions, which are stable in 1 with winding number $N=0$, and unstable in 2 with $N=2$. In region 3 the solutions are slow and unstable, $N=1$. The inset is an enlargement of the graph near the point o, where the curve corresponding to the Hopf bifurcation locus intersects with the curve for the extinction locus.

However, they do not cross the imaginary axis at $c=c_{\mathrm{e}}$ but they hit the origin along the imaginary axis instead, giving birth to a pair of purely real eigenvalues. One of these eigenvalues moves to the left half-plane, while the other to the right half-plane, as we decrease $c$ further. The Hopf bifurcation ceases to exist for $l>l_{\mathrm{o}}$. In this case a point of the discrete spectrum moves along the real axis from the left half-plane to the right half-plane as we pass from the fast to the slow branch of the solutions. The scenario we have just outlined qualitatively agrees with predictions of the MAE described in $\S 5$.

In figure 9 we plot the critical parameter $c$ as a function of $l$ for both the Hopf bifurcation and extinction. The graph is drawn for different values of $\tau=0.1,0.2,0.3$, and 0.4. As we increase $l$, the region where an oscillatory instability manifests itself is squeezed out towards the larger values of heat loss. In figure $9 a$ we plot the dependence of the front speed $c_{\mathrm{o}}$, where the curves for the Hopf bifurcation and the extinction intersect, as a function of $l$. The function $c_{\mathrm{o}}(l)$ obtained from the matched asymptotic analysis is also drawn on the same graph. As we can see, the difference between the numerical results and the prediction of the asymptotic method is substantial. This is expected, since the linear-stability analysis outlined in $\S 5$ is valid for $\tau$ asymptotically close to unity. However, for $\tau \sim 1$ the value of $l_{\mathrm{o}}$ tends to zero very rapidly and it is difficult to verify the asymptotic dependence of $c_{\mathrm{o}}(\tau)$ and $l_{\mathrm{o}}(\tau)$ numerically.

In figure 10, the Hopf frequency $\omega$ as a function of $l$ is plotted for $\tau=0.1,0.2,0.3$, and 0.4 . We also present the values of $\omega$ in the adiabatic case $(l=0)$, which were obtained in Gubernov et al. (2003), by the dashed lines in the left part of the graph. The convergence to the adiabatic values is fast for $\tau=0.1$ and 0.2 , whereas it manifests itself for smaller values of $l$ in the case $\tau=0.3$ and 0.4 . In the right part of the figure all curves show the rapid decay of $\omega$. This occurs when we approach 


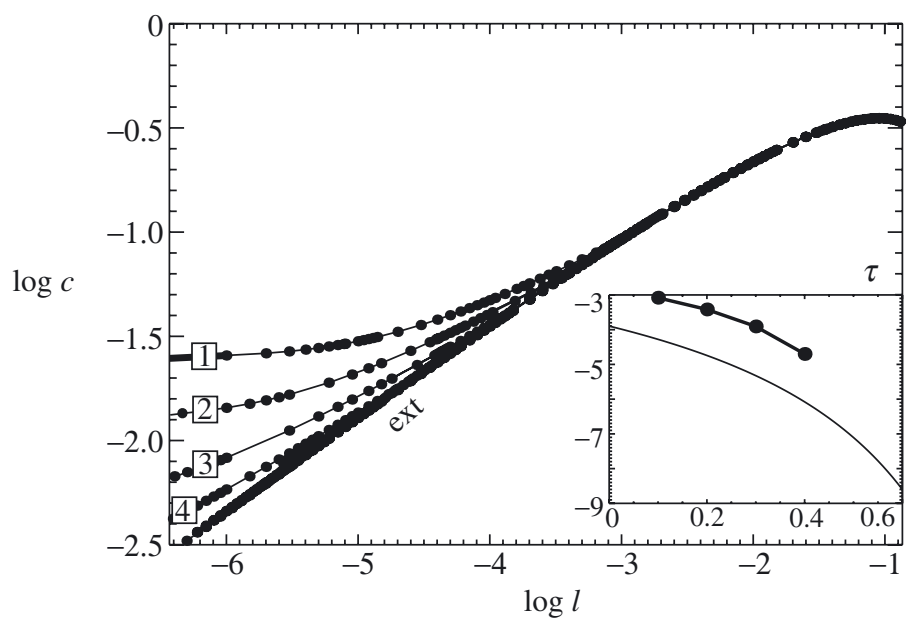

Figure 9. Critical parameter $c$ versus $l$ for $u_{\mathrm{a}}=0$. Dots connected with solid lines correspond to numerical results. Curves 1, 2, 3 and 4 correspond to the critical parameters of the Hopf bifurcation for $\tau=0.1,0.2,0.3$ and 0.4 , respectively. Curves labelled with 'ext' depict the extinction limit for the same set of $\tau$ values. In the inset the dependence $c_{\mathrm{o}}$ on $\tau$ is plotted; dots connected with lines denote the numerical results, solid line represents the prediction of the asymptotic analysis.

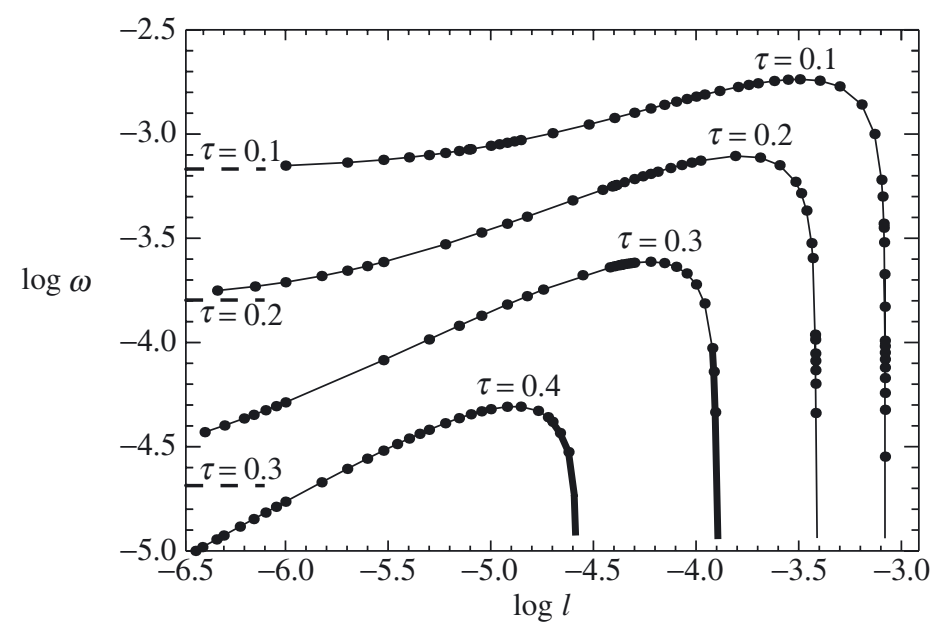

Figure 10. Hopf frequency $\omega$ as a function of $l$ for $u_{\mathrm{a}}=0$ and $\tau=0.1,0.2,0.3$ and 0.4.

Dashed lines on the left correspond to the adiabatic $(l=0)$ values of $\omega$.

the point where the Hopf and the extinction loci intersect and the two points of the discrete spectrum hit the origin along the imaginary axis, which results in the Hopf frequency $\omega \rightarrow 0$.

(b) Non-zero ambient temperature $\left(u_{\mathrm{a}}>0\right)$

Next we generalize the results of the previous subsection for the case of $u_{\text {a }}$ greater than zero.

Proc. R. Soc. Lond. A (2004) 


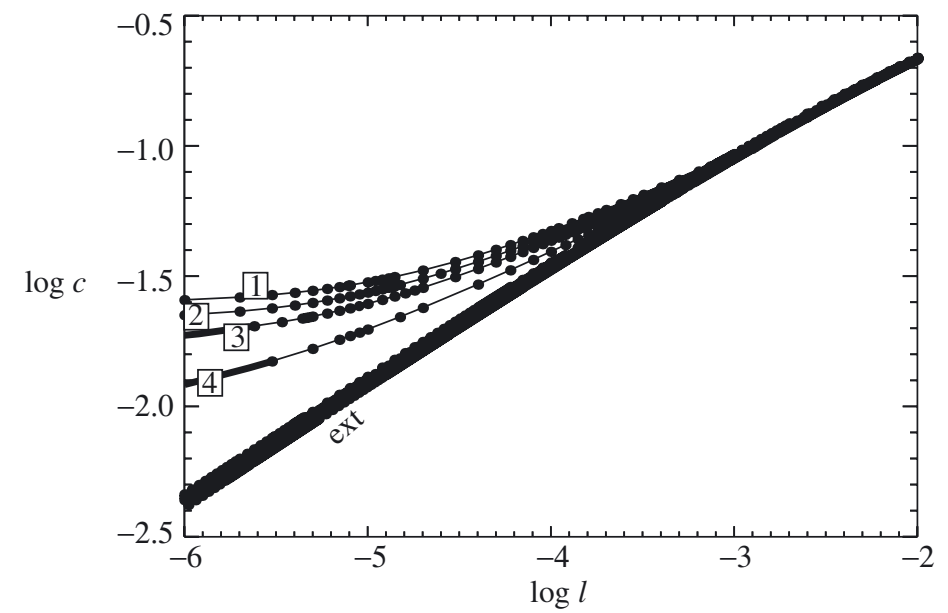

Figure 11. Critical parameter $c$ versus $l$ for $\tau=0.1$. Dots connected with solid lines represent numerical values. Curves 1, 2, 3 and 4 correspond to the critical parameters of the Hopf bifurcation for $u_{\mathrm{a}}=0,0.005,0.01$ and 0.02 , respectively. Curves labelled with 'ext' depict the extinction limit for the same set of $u_{\mathrm{a}}$ values.

In figure 11 we show the critical parameter $c$ as a function of $l$ for both the Hopf bifurcation and extinction. The graph is plotted for fixed $\tau$ and different values of $u_{\mathrm{a}}=0,0.005,0.01$ and 0.02 . In figure 12, the Hopf frequency $\omega$ as a function of $l$ is plotted for $\tau=0.1$ and different values of the ambient temperature. As can be seen from both figures the inclusion of the non-zero ambient temperature into the model does not change its qualitative behaviour. However, if one is interested in quantitative results, for example, in order to compare the prediction of the model with experimental data, taking $u_{\mathrm{a}}$ into consideration is essential.

\section{Conclusions}

In this paper we have considered the model with one-step chemistry for the propagation of the non-adiabatic combustion fronts for the cases with both zero and non-zero ambient temperature.

We have numerically investigated the stationary solutions using the shooting and relaxation methods. For the case of $u_{\mathrm{a}}=0$ the results of this paper substantially extend the analysis carried out in our previous paper (Gubernov et al. 2003), where the heat loss was neglected. The comparison of the model with the heat loss and without it reveals the fact that the difference in the characteristic coordinate length in which the solution changes significantly in the preheat, reaction and product zones is much more substantial in the non-adiabatic case. Therefore, the numerical algorithms have been modified to take into account this difference by using the non-uniform coordinate grid. This then allows us to investigate the properties of the stationary solutions such as the dependence of the extinction limit, the speed of the front and the residual amount of fuel left behind the front for a wide range of parameter values. The numerical results were compared with the prediction of the MAE method. The difference between the numerical data and the prediction of asymptotic analysis for the speed of the front and the extinction limit is substantial for small and moderate values of $\beta$, and it becomes less significant for large values 


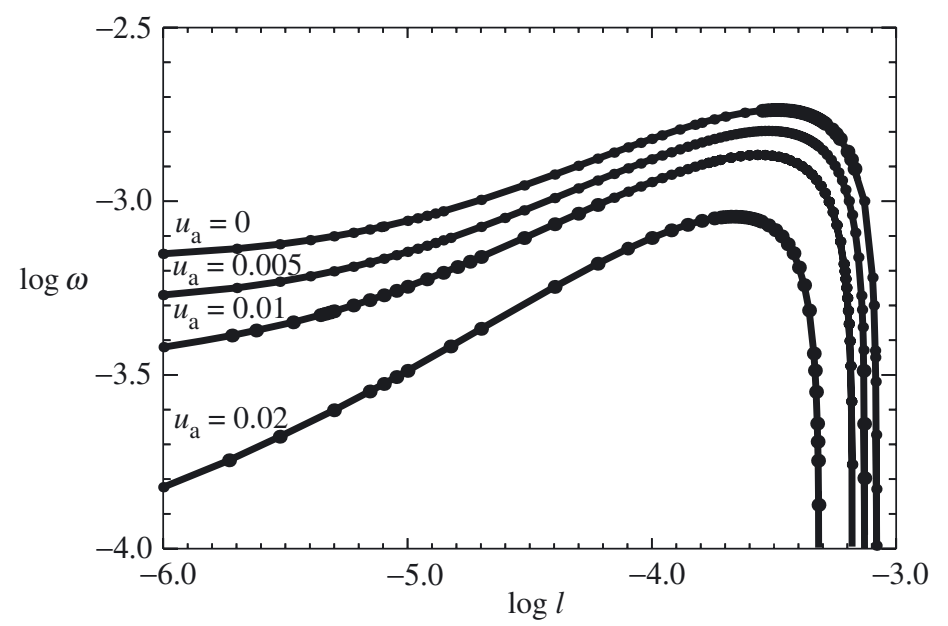

Figure 12. Hopf frequency $\omega$ as a function of $l$ for $\tau=0.1$ and $u_{\mathrm{a}}=0,0.005,0.01,0.02$.

of $\beta$, especially when $\tau=1$. This is expected as the MAE is valid for large values of $\beta$ and higher-order terms of the expansion have to be considered to give a better correspondence with the numerical results.

Next we generalized the model to allow non-zero ambient temperature. The behaviour of the system with $u_{\mathrm{a}}>0$ is qualitatively the same as for the case of zero ambient temperature. However, the investigation reveals some quantitative differences between these two cases. In particular we showed that increasing $u_{\mathrm{a}}$ results in the growth of the region of parameter values for which the travelling-wave solution exists.

The linear-stability problem for the stationary solution has also been considered. We solved it numerically using the Evans function approach, which is extended by the compound-matrix method. The details of the algorithm are described in our previous paper (Gubernov et al. 2003). In the present paper we extend the applicability of the methods to non-uniform grids. We were able to provide a detailed description of the scenarios of transition to instability as the parameters of the problem are varied. It was shown that there are two main ways for the onset of instability in the system. Firstly, if the heat loss is less than the critical value $l_{\mathrm{o}}$, then for fixed $\tau$ the steady propagating front exhibits an oscillatory instability via the Hopf bifurcation, as we move from the fast to the slow branch along the family of the stationary problem solutions $c(\beta, \tau, l)$. On the other hand, if $l>l_{\mathrm{o}}$, then for fixed $\tau$ the fast branch is stable and the slow branch is unstable; the transition to instability occurs at the point of extinction in a monotonous way. The critical values for the Hopf bifurcation and the extinction were found in the parameter space for small and moderate values of $\tau$. The results were compared with the prediction of the matched asymptotic analysis in the case of zero ambient temperature. It was shown that in the range of parameters considered in this paper, the asymptotic analysis gives the correct qualitative description of the transition to instability.

The authors are thankful to A. R. Champneys, S. J. Malham, D. E. Pelinovsky and the referees for helpful comments.

Proc. R. Soc. Lond. A (2004) 


\section{References}

Afendikov, A. L. \& Bridges, T. J. 2001 Instability of the Hocking-Stewartson pulse and its implications for three-dimensional Poiseuille flow. Proc. R. Soc. Lond. A 457, 1-16.

Bayliss, A. \& Matkowsky, B. J. 1990 Two routes to chaos in condensed-phase combustion. SIAM J. Appl. Math. 50, 437-459.

Billingham, J. \& Mercer, G. N. 2001 The effect of heat loss on the propagation of strongly exothermic combustion waves. Combust. Theory Model. 5, 319-342.

Booty, R. O., Margolis, S. B. \& Matkowsky, B. J. 1987 Interaction of pulsating and spinning waves in non-adiabatic flame propagation. SIAM J. Appl. Math. 47, 1241-1286.

Brailovsky, I. \& Sivashinsky, G. 1993 Chaotic dynamics in solid-fuel combustion. Physica D 65, 191-198.

Bush, W. B. \& Fendell, F. E. 1970 Asymptotic analysis of laminar flame propagation for general Lewis numbers. Combust. Sci. Technol. 1, 421-428.

Frankel, M., Roytburd, V. \& Sivashinsky, G. 1994 A sequence of period doublings and chaotic pulsations in a free boundary problem modelling thermal instabilities. SIAM J. Appl. Math. 54, 1101-1112.

Gubernov, V., Mercer, G. N., Sidhu, H. S. \& Weber, R. O. 2003 Evans function stability of combustion waves. SIAM J. Appl. Math. 63, 1259-1275.

Henry, D. 1981 Geometric theory of semilinear parabolic equations. Springer.

Joulin, G. \& Clavin, P. 1979 Linear stability analysis of non-adiabatic flames: diffusional thermal model. Combust. Flame 35, 139-153.

Kuznetsov, Y. A. 1981 Elements of applied bifurcation theory. Springer.

Makino, A. 2001 Fundamental aspects of the heterogeneous flame in the self-propagating hightemperature synthesis (SHS) process. Prog. Energy Combust. Sci. 27, 1-74.

Margolis, S. B. 1991 The transition to non-steady deflagration in gasless combustion. Prog. Energy Combust. Sci. 17, 135-162.

Margolis, S. B. \& Matkowsky, B. J. 1983 Nonlinear stability and bifurcation in the transition from laminar to turbulent flame propagation. Combust. Sci. Technol. 34, 45-77.

Matkowsky, B. J. \& Olagunju, D. O. 1980 Propagation of a pulsating flame front in a gaseous combustible mixture. SIAM J. Appl. Math. 39, 290-300.

Matkowsky, B. J. \& Sivashinsky, G. I. 1978 Propagation of a pulsating reaction front in solid fuel combustion. SIAM J. Appl. Math. 35, 465-478.

Mercer, G. N., Weber, R. O. \& Sidhu, H. S. 1998 An oscillatory route to extinction for solid-fuel combustion waves due to heat losses. Proc. R. Soc. Lond. A 454, 2015-2022.

Merzhanov, A. G. \& Rumanov, E. N. 1999 Physics of reaction waves. Rev. Mod. Phys. 71, 1173-1211.

Moore, J. J. \& Feng, H. J. 1995 a Combustion synthesis of advanced materials. I. Reaction parameters. Prog. Mater. Sci. 39, 243-273.

Moore, J. J. \& Feng, H. J. $1995 b$ Combustion synthesis of advanced materials. II. Classification, applications and modelling. Prog. Mater. Sci. 39, 275-316.

Press, W. H., Teukolsky, S. A., Vetterling, W. T. \& Flannery, B. P. 1992 Numerical recipes in $C$ : the art of scientific computing. Cambridge University Press.

Sandstede, B. 2002 Stability of travelling waves. In Handbook of dynamical systems II (ed. B. Fiedler), pp. 983-1055. Elsevier.

Schult, D. A. 1999 Matched asymptotic expansions and the closure problem for combustion waves. SIAM J. Appl. Math. 60, 136-155.

Shkadinskii, K. G., Khaikin, B. I. \& Merzhanov, A. G. 1971 Propagation of the pulsating exothermic reaction front in the condensed phase. Combust. Expl. Shock Waves 7, 15-22.

Proc. R. Soc. Lond. A (2004) 
Volpert, A. I., Volpert, V. A. \& Volpert, V. A. 1994 Traveling-wave solutions of parabolic systems. Translations of Mathematical Monographs, vol. 140. Providence, RI: American Mathematical Society.

Watt, S. D., Weber, R. O., Sidhu, H. S. \& Mercer, G. N. 1999 A weight-function approach for determining watershed initial conditions for combustion waves. IMA J. Appl. Math. 62, 195-206.

Weber, R. O., Mercer, G. N., Sidhu, H. S. \& Gray, B. F. 1997 Combustion waves for gases $(L e=1)$ and solids $(L e \rightarrow \infty)$. Proc. R. Soc. Lond. A 453, 1105-1118.

Weber, R. O., Balakrishnan, E. \& Wake, G. C. 1998 Critical initial conditions for spontaneous thermal ignition. J. Chem. Soc. Faraday Trans. 94, 3613-3617. 